\title{
AJUSTE PSICOSOCIAL Y ESTADO DEPRESIVO EN ADOLESCENTES DE CENTROS ESCOLARES DE LIMA METROPOLITANA
}

\author{
Mario S. Bulnes B., Carlos R. Ponce D., Rosa E. Huerta R., Roger Elizalde B., Willy R. Santiváñez O.,
} Jaime R. Aliaga T., Leni Álvarez T., Roberto F. Calmet A.

\begin{abstract}
RESUMEN
A partir de la preocupación por el aumento de la frecuencia de depresión y la disminución de la edad de las personas que padecen este tipo de problema, así como algunos indicadores que lo acompañan y que se encuentra dentro del concepto de desajuste psicosocial, surge la necesidad de estudiar dichos fenómenos en la población escolar.

Objetivos: Demostrar la relación interdependiente de las variables ajuste psicosocial y depresión en escolares del nivel secundario de Lima Metropolitana, en función al tipo de colegio (estatal y particular) y género. Diseño: Es una investigación de tipo básica y de diseño descriptivo comparativo y correlacional. Material y método: La muestra estuvo constituida por 480 escolares de centros educativos estatales y particulares, cuyas edades oscilan entre los 12 y 17 años. Para la evaluación de la variable ajuste psicosocial, se utilizó el Inventario de Adaptación de Conducta de M. Victoria de la Cruz y Agustín Cordero, y para evaluar la variable depresión, se utilizó el Cuestionario de Depresión para niños y adolescentes de M. Lang y M. Tisher. El análisis estadístico derivó en primer lugar, en un análisis de validez y confiabilidad de las pruebas, y en segundo lugar, se realizó un análisis inferencial para la contrastación de las hipótesis planteadas. Resultados: Se ha encontrado que las dos pruebas utilizadas son altamente válidas y confiables.
\end{abstract}

Palabras claves: Ajuste psicosocial, depresión, realización, bienestar.

\begin{abstract}
From the preoccupation by the increase of the depression frequency and the diminution of the age of the people who suffer this type of problem, as well as some indicators that accompanies it and that is within the concept of psycho-social misalignment, arise the necessity to study these phenomena in the scholastic population. Objectives: demostrate the interdependent relation of the variable adjustment psycho-social and depression in students of the city of Lima, Peru, in function to the type of school (state and private) and sort. Design: Descriptive comparative and correlational. Materials and methods: The sample was constituted by 480 students of state and particular educative centers, whose ages oscillate between the 12 and 17 years. For the evaluation of the variable psychosocial adjustment, the Inventory of Adaptation of Conduct of M. Victoria de la Cruz and Agustín Cordero was used, and to evaluate the variable depression, it was used the Questionnaire of Depression for children and adolescents of M. Lang and M. Tisher. The statistical analysis derived in the first place, in an analysis of validity and trustworthiness of the tests, and secondly, a
\end{abstract}


inferencial analysis for the contrast of the raised hypotheses was made. Results: One has been that the two used tests are highly valid and reliable.

Keywords: Psychosocial adjustment, depression, realization, welfare.

\section{INTRODUCCIÓN}

La adolescencia es un periodo del desarrollo que se caracteriza por producirse grandes cambios en la formación del individuo, los cuales se traducen en trasformaciones físicas, definición de las características más importantes de la personalidad y la asunción de nuevos roles sociales, desarrollándose de esta forma, habilidades y capacidades fundamentales para afrontar la vida adulta.

Este periodo es asumido como una etapa de tránsito que puede ser más o menos traumática, debido básicamente a que un porcentaje muy elevado de los problemas humanos se inician en este ciclo vital, los que de no abordarse en forma inmediata, pueden mantenerse y agravarse posteriormente. En este sentido, como refiere Saldaña (2001) ${ }^{1}$, la familia, la escuela y los amigos son los referentes más importantes que tienen los adolescentes durante este periodo, los cuales pueden ser tanto protectores como favorecedores de la aparición de situaciones de desajuste psicosocial, y por lo tanto, pueden ser considerados dentro de las actividades de prevención de los mismos.

Sin embargo, todo indica que existen conductas observables en la adolescencia que no son bien canalizadas en los espacios de socialización, como la familia y la escuela, ni se producen cambios significativos en su actividad cotidiana, como los estudios, tareas, tiempo libre y de comunicación, obstaculizando de esta forma, el logro de la autonomía que el adolescente tanto desea, generando con ello inseguridad personal, desajuste y depresión ante el nuevo rol social que ocupa, el cual es etiológicamente hablando de naturaleza multicausal.

En otras palabras, se debe tener en cuenta que la vida, en un sentido amplio, puede definirse como un proceso permanente de cambio y adaptación, en el que se pasa de un estado inicial de dependencia hacia otro de autonomía e independencia, y en ella, el factor social más influyente y que mejor define a nuestra sociedad a inicios del siglo XXI, es su cada vez mayor tendencia hacia la complejidad, volviéndose altamente competitiva, individualista y no solidaria.

En este contexto, los adolescentes participan en un determinado sistema social que provee sus normas y valores, a los que cada uno otorgará una significación muy vinculado a las historias personales y las creencias del contexto en los que vive. Ciertos factores desmoralizadores en el entorno social y la experiencia pasada, tornan al individuo más vulnerable a los hechos vitales. Al respecto, un dato significativo es el que menciona Ponce $(2003)^{2}$, quien encuentra en una población de escolares de Lima de diferentes estratos socioeconómicos, que los problemas de desajuste son más significativos en los estratos altos en comparación a otros estratos, demostrando con esto, que el desajuste psicosocial, más que un problema de pobreza, es un problema de convivencia y de estilo de vida.

En cuanto a la depresión, Berry, J. (1992) $)^{3}$ hace referencia a que la frecuencia con la que se presenta ha aumentado, mientras que la edad de iniciación ha disminuido, por lo que se está convirtiendo en un fenómeno frecuente el observar la presencia de depresión en menores de edad, resultando desalentador el empezar a percibir que este problema deteriora 
significativamente la adaptación psicosocial y el desarrollo de la personalidad de los adolescentes. Por otro lado, estudios longitudinales prueban que existe una continuidad entre los problemas padecidos en la niñez y adolescencia y los que se sufren en la edad adulta, estos estudios también refieren al aumento de riesgo de depresión en niños y adolescentes con historias familiares que reconocen la presencia de trastorno depresivo (Saldaña, 2001). Este vínculo familiar se encuentra asociado con situaciones de estrés psicosocial que afectan a niños y adolescentes con cierto margen de vulnerabilidad (muertes, abandonos, crisis económicas, inestabilidad laboral) que deben ser atendidos.

Por otro lado, es alarmante el aumento de suicidios y actos de violencia en menores de edad, que amenaza convertir la muerte de niños y adolescentes en algo cotidiano. En este sentido, es necesario resaltar que estos actos conforman algunas de las diversas formas en las cuales se expresa una intensa frustración mal canalizada y las dificultades que tiene para encausar su comportamiento, el cual debiera estar dirigido al logro de su realización y bienestar personal, sin embargo, al poner en peligro su integridad personal, constituye por el contrario en indicadores de desajuste, en tanto que los recursos personales con los que cuenta y que son producto de su interacción familiar y escolar, son insuficientes para hacerle frente a dichas dificultades cotidianas (Arenas, 1998) ${ }^{4}$.

De todo esto, se concluye que la vida moderna ha producido muchos cambios en los modelos de vida familiar, y ha afectado sustancialmente las relaciones en la familia, por ejemplo, se trabaja menos en casa, hay más esposas que trabajan fuera del hogar, el divorcio y las separaciones son más corrientes, la educación y formación se realizan más en la escuela, las diversiones han huido fuera del hogar, la movilidad social y vocacional ha aumentado, el medio material del hogar se ha vuelto más importante (Saldaña, 2001).

Según Casullo, Bonaldi y Fernández $(2000)^{5}$, se ha observado que en niños y adolescentes con síntomas depresivos se encuentran asociados al pobre desarrollo de las habilidades sociales, bajo autoconcepto, e importantes dificultades para poder estudiar y aprender, es más, también refieren que existen estudios en los que se afirman que la presencia de síntomas depresivos aumenta con el paso de la preadolescencia a la adolescencia y que dicho aumento era mayor entre las mujeres en comparación a los varones.

\section{Planteamiento del problema}

El presente estudio pretende responder a las siguientes interrogantes:

1. ¿Existe relación entre la variable Ajuste Psicosocial y la variable Depresión, en escolares de centros escolares estatales y particulares de Lima Metropolitana?

2. ¿Existe diferencias significativas en las variables Ajuste Psicosocial y la variable Depresión, en escolares de Lima Metropolitana, según el género y tipo de colegio (estatal y particular)?

\section{Objetivos}

1. Analizar la relación que existe entre las variables Ajuste Psicosocial y Depresión en adolescentes de centros escolares de Lima Metropolitana.

2. Comparar la variable Ajuste Psicosocial, en adolescentes, según el tipo de colegio. 
3. Comparar la variable Depresión, en adolescentes, según el tipo de colegio.

4. Comparar la variable Ajuste Psicosocial, en adolescentes, según el género y tipo de colegio.

5. Comparar la variable Depresión, en adolescentes, según el género y tipo de colegio.

\section{Hipótesis}

1. Existe una correlación significativa entre las variables Ajuste Psicosocial y Depresión, en adolescentes de centros escolares de Lima Metropolitana.

2. Existen diferencias estadísticamente significativas en la variable Ajuste Psicosocial, en adolescentes de centros escolares de Lima Metropolitana, según el tipo de colegio.

3. Existen diferencias estadísticamente significativas en la variable Depresión, en adolescentes de centros escolares de Lima Metropolitana, según el tipo de colegio.

4. Existen diferencias estadísticamente significativas en la variable Ajuste Psicosocial, en adolescentes de centros escolares de Lima Metropolitana según, el género y tipo de colegio.

5. Existen diferencias estadísticamente significativas en la variable Depresión, en adolescentes de centros escolares de Lima Metropolitana, según el género y tipo de colegio.

\section{MATERIAL Y MÉTODO}

Se utilizó el diseño descriptivo correlacional y comparativo, en la medida que se trata de describir y luego correlacionar ambas variables, motivo de estudio, para luego comparar los resultados según el género y tipo de centro escolar (Sánchez y Reyes, 2002) ${ }^{6}$.

El universo de investigación estuvo conformado por los alumnos matriculados en primer, segundo, tercer, cuarto y quinto año de secundaria de Lima Metropolitana. La muestra de estudio estuvo conformada por 482 escolares, de los cuales 311 correspondían a centros escolares estatales, y 171 a centro escolares particulares de Lima Metropolitana, cuyas edades oscilan entre los 12 y 17 años, entre hombres y mujeres, y cuyos grados de estudio están comprendidos entre los cinco grados de secundaria, tal como se puede observar en las tablas 1 y 2. 
Tabla 1. Composición de la muestra según tipo de colegio y sexo.

\begin{tabular}{|c|c|c|c|}
\hline Tipo de colegio & Sexo & $\mathbf{F}$ & Total \\
\hline \multirow{2}{*}{ Estatal } & Masculino & $\begin{array}{c}145 \\
46.62 \% \\
\end{array}$ & \multirow{2}{*}{$\begin{array}{c}311 \\
99.99 \%\end{array}$} \\
\hline & Femenino & $\begin{array}{c}166 \\
53.37 \%\end{array}$ & \\
\hline \multirow{2}{*}{ Particular } & Masculino & $\begin{array}{c}99 \\
57.89 \% \\
\end{array}$ & \multirow{2}{*}{$\begin{array}{c}171 \\
99.99 \%\end{array}$} \\
\hline & Femenino & $\begin{array}{c}72 \\
42.10 \% \\
\end{array}$ & \\
\hline
\end{tabular}

Tabla 2. Composición general de la muestra según grado escolar y tipo de colegio.

\begin{tabular}{|c|c|c|c|}
\hline \multirow{2}{*}{ Grado escolar } & \multicolumn{2}{|c|}{ Tipo de colegio } & \multirow{2}{*}{ Total } \\
\hline & Estatal & Particular & \\
\hline \multirow{2}{*}{ Primer año } & 91 & 23 & 114 \\
\hline & $29.26 \%$ & $13.45 \%$ & $23.65 \%$ \\
\hline \multirow{2}{*}{ Segundo año } & 69 & 35 & 104 \\
\hline & $22.18 \%$ & $20.46 \%$ & $21.58 \%$ \\
\hline \multirow{2}{*}{ Tercer año } & 19 & 56 & 75 \\
\hline & $6.12 \%$ & $32.74 \%$ & $15.56 \%$ \\
\hline \multirow{2}{*}{ Cuarto año } & 75 & 31 & 106 \\
\hline & $24.11 \%$ & $18.12 \%$ & $21.99 \%$ \\
\hline \multirow{2}{*}{ Quinto año } & 57 & 26 & 83 \\
\hline & $18.32 \%$ & $15.22 \%$ & $17.22 \%$ \\
\hline \multirow{2}{*}{ Total } & 311 & 171 & 482 \\
\hline & $100.00 \%$ & $100.00 \%$ & $100 \%$ \\
\hline
\end{tabular}

\section{Variables de estudio}

a) Independiente:

Centros escolares estatales y particulares.

b) Dependiente:

Resultados de las pruebas aplicadas, para lo cual se utilizó el Inventario de Adaptación de Conducta, de M. Victoria de la Cruz y Agustín Cordero, y el Cuestionario de Depresión para niños y adolescentes, de M. Lang y M. Tiher. 


\section{Técnicas e instrumentos de recolección de datos}

Para la evaluación de las variables estudiadas, se utilizaron los siguientes instrumentos psicológicos:

a) Inventario de Adaptación de Conducta (IAC): Es un instrumento elaborado por M. Victoria de la Cruz y Agustín Cordero (1981) ${ }^{7}$. El IAC es un inventario formado por 123 ítemes y evalúa las siguientes dimensiones: Ajuste personal, ajuste familiar, ajuste escolar, ajustes social.

Es una prueba altamente confiable, confirmado por el Alpha de Cronbach, obtenido por los autores de la prueba, el cual alcanza un coeficiente de .90 , que en nuestro caso es de .9066 .

b) Cuestionario de Depresión para niños y adolescentes: Elaborado por M. Lang y M. Tisher $(1990)^{8}$. Esta prueba mide dos grandes dimensiones de la depresión: Total depresivo y Total positivo. Cada una de ellas está conformada por un determinado número de subescalas, las cuales se detallan a continuación:

\section{Total depresivo (66 ítemes)}

Respuesta afectiva, problemas sociales, autoestima, preocupación por la muerte, sentimiento de culpabilidad, depresivos varios.

\section{Total positivo (48 ítemes)}

Ánimo-alegría, positivo varios

En el presente estudio, se obtuvo un Alpha de Crombach de .9059, lo cual permite confirmar el buen nivel de estabilidad y consistencia interna de la prueba.

\section{Técnicas de procesamiento y análisis de datos}

a) Análisis descriptivo y exploratorio: Frecuencia, porcentajes, media, desviación standard.

b) El análisis inferencial para la contrastación de hipótesis.

c) Análisis psicométrico de las pruebas: Análisis de ítemes con el cómputo de las correlaciones ítem-test, y la confiabilidad, cálculo de los coeficientes Alfa de Cronbach.

d) Correlación de los factores (Pearson).

\section{RESULTADOS}

Los resultados serán presentados de acuerdo a la siguiente secuencia:

a) Análisis exploratorio de las variables de estudio.

b) Análisis de correlación de las variables motivo de estudio.

c) Análisis comparativo en función al tipo de colegio.

d) Análisis comparativo en función al género. 
a) Análisis exploratorio de las variables de estudio (Tabla 3).

Como se puede observar, la media en la variable Adaptación de Conducta, es mayor en escolares de colegios particulares, lo que revela un mejor nivel de ajuste en relación a los escolares de colegios estatales.

En la variable Depresión se observa una media mayor en escolares de colegios estatales en relación a los escolares de colegios particulares.

Tabla 3. Media y desviación estándar de las variables, adaptación de conducta y depresión en escolares de colegios estatales y particulares.

\begin{tabular}{|c|c|c|c|c|}
\hline \multirow{2}{*}{ Variables } & \multicolumn{2}{|c|}{ Colegio estatal } & \multicolumn{2}{c|}{ Colegio particular } \\
\cline { 2 - 5 } & Media & D.S. & Media & D.S. \\
\hline $\begin{array}{c}\text { Adaptación de } \\
\text { conducta }\end{array}$ & 70.05 & 15.749 & 74.93 & 16.767 \\
\hline Depresión & 134.84 & 30.599 & 117.80 & 31.087 \\
\hline
\end{tabular}

\section{b) Análisis de correlación de las variables}

\section{b.1. Análisis de correlación de las variables adaptación de conducta y depresión, en} escolares de colegios estatales (Tabla 4).

Al analizar los resultados correspondientes al coeficiente de correlación Spearman, se obtiene una correlación negativa y significativa de -.357 en su conjunto, como un todo. Esto se repite cuando se analiza la correlación de la variable adaptación de conducta en su puntaje total con los subtests de la variable Depresión, y viceversa. Pero al analizar la correlación de los subtests de las pruebas entre sí, por separado, si bien también se mantiene la correlación significativa en algunos subtests, también se observan algunas bajas correlaciones o correlaciones no significativas, como por ejemplo:

- Adaptación social - Respuesta afectiva, en la cual se obtiene una correlación de -.032. De esto se puede inferir que la adaptación social tiene pocas implicancias con las respuestas afectivas.

- Adaptación social - Problemas sociales, en el cual se obtiene una correlación de -.075. Se debe entender que el subtest de la variable Depresión correspondiente a problemas sociales, hace referencia al aislamiento y soledad del niño, que en este caso, debe ser interpretado como que la adaptación social no tiene implicancias con los problemas sociales mencionados.

- Adaptación social - Preocupación por la muerte, en el cual se alcanza una correlación de -.003, con lo que se puede decir, a raíz de los puntajes alcanzados, que dichos subtests conforman variables autónomas.

- Adaptación social - Sentimiento de culpa, alcanzando una correlación de .031, de igual manera no se observa ninguna implicancia entre la adaptación social y el subtest sentimiento de culpa. 
Tabla 4. Correlación entre los factores de la adaptación de conducta y los factores de la depresión en escolares de colegios estatales.

\begin{tabular}{|c|c|c|c|c|c|c|c|c|c|}
\hline Depresión & $\begin{array}{l}\text { Ánimo - } \\
\text { Alegría }\end{array}$ & $\begin{array}{c}\text { Respuesta } \\
\text { afectiva }\end{array}$ & $\begin{array}{l}\text { Problemas } \\
\text { sociales }\end{array}$ & Autoestima & $\begin{array}{c}\text { Preocupación } \\
\text { por la } \\
\text { muerte }\end{array}$ & $\begin{array}{l}\text { Sentimiento } \\
\text { de culpa }\end{array}$ & $\begin{array}{c}\text { Depresivos } \\
\text { varios }\end{array}$ & $\begin{array}{c}\text { Positivos } \\
\text { varios }\end{array}$ & $\begin{array}{c}\text { Total } \\
\text { depresivo }\end{array}$ \\
\hline Personal & $-.382 *$ & $-.435 *$ & $-.454 *$ & $-.514 *$ & $-.420 *$ & $-.360 *$ & $-.308 *$ & $-.253 *$ & $.800 *$ \\
\hline Familiar & $-.354 *$ & $-.288 *$ & $-.341 *$ & $-.354 *$ & $-.218 *$ & $-.208 *$ & -.175 * & $-.377 *$ & $.796 *$ \\
\hline Escolar & $-.133 *$ & -.225 * & -.215 * & $-.251 *$ & -.193 * & -.115 * & -.095 & -.089 & $.784 *$ \\
\hline Social & $-.344 *$ & -.032 & -.075 & $-.125 *$ & -.003 & .031 & .105 & $-.280 *$ & $.584 *$ \\
\hline $\begin{array}{c}\text { Total } \\
\text { adaptación }\end{array}$ & $-.398 *$ & $-.333 *$ & $-.367 *$ & $-.419 *$ & $-.282 *$ & $-.222 *$ & $-.164 *$ & $-.331 *$ & $-.357 *$ \\
\hline
\end{tabular}

$$
\begin{gathered}
* \mathrm{p}<.05 \\
\mathrm{n}=311
\end{gathered}
$$




\section{b.2. Análisis de correlación de las variables adaptación de conducta y depresión, en escolares de colegios particulares (Tabla 5).}

$\mathrm{Al}$ analizar los resultados correspondientes, en su conjunto como un todo, se obtiene una correlación negativa y significativa de -.582. Esto se repite cuando se analiza la correlación de la variable adaptación de conducta en su puntaje total con los subtests de la variable Depresión, y viceversa, tan igual como en el análisis anterior de los resultados correspondientes a la tabla 4, pero con algunas variantes, como por ejemplo:

- Adaptación social - Respuesta afectiva, a diferencia de los escolares de colegios estatales, en la muestra de escolares de colegios particulares sí existe implicancia entre la adaptación social y las respuestas afectivas (-.305).

- Adaptación social - Problemas sociales, de igual manera a diferencia de los escolares de colegios estatales, en la muestra de escolares de colegios particulares sí existe implicancia entre la adaptación social y los problemas sociales de la prueba de Depresión, vale decir que de haber problemas de adaptación social se visualizaría en una tendencia al aislamiento y soledad del adolescente (-.266).

- Adaptación social - Preocupación por la muerte - Sentimiento de culpa, en este aspecto las características de acuerdo a dichos subtests se mantienen igual a la muestra anterior, -.111 y -.056 respectivamente.

\section{Contrastación de hipótesis}

Al analizar la primera hipótesis (H1) en la que se correlaciona la variable Adaptación de Conducta y la variable Depresión, tanto en escolares de colegios estatales como de colegios particulares por separado, se encontró que había una correlación significativa en ambos grupos $(\mathrm{r}=-.357, \mathrm{y} \mathrm{r}=-.582$ respectivamente), confirmando la primera hipótesis. 
Revista de Investigación en Psicología - Vol. 8, N. ${ }^{\circ} 2$

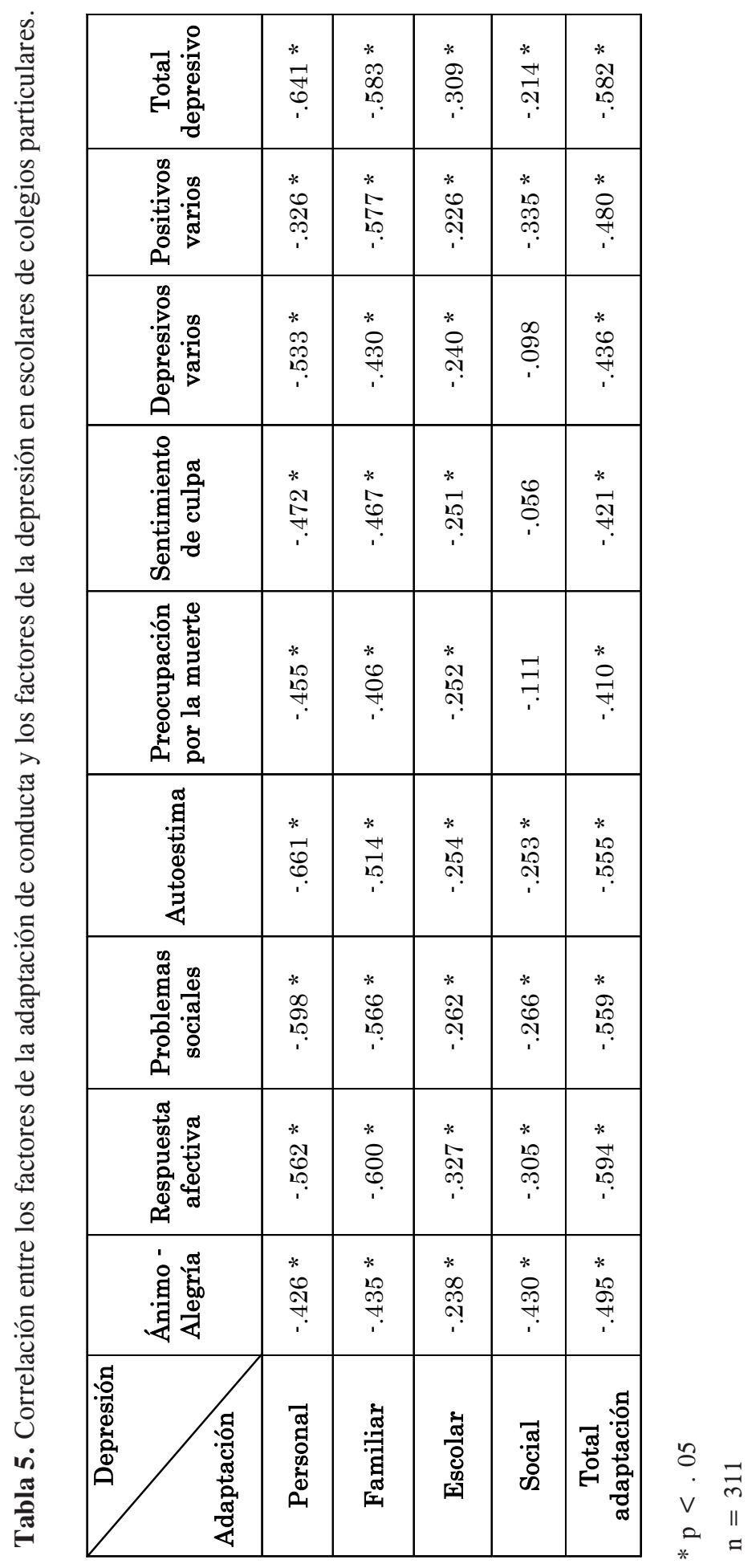




\section{c) Análisis comparativo en función al tipo de colegio}

\section{c.1. Análisis de las comparaciones según la variable adaptación de conducta, en escolares} de colegios estatales y particulares (Tabla 6)

Al realizar el análisis correspondiente a la variable Adaptación de Conducta, en ambas muestras de estudio, se observaron diferencias estadísticamente significativas, obteniendo un $\mathrm{t}=-3.177$, tomando como referencia el puntaje total de la prueba. Al establecer los detalles de dicho resultado, se obtuvo que las mayores diferencias se encuentran en los subtests Adaptación Personal, Adaptación Familiar y Adaptación Social, por lo que se puede interpretar que los escolares de colegios estatales enfrentan una mayor dificultad en la adaptación en esas áreas. En este sentido, se puede mencionar que son probablemente más vulnerables en cuanto a su autoestima, en su convivencia familiar y en el campo social.

Sin embargo, en el subtest Adaptación Escolar no se encuentran diferencias estadísticamente significativas $(\mathrm{r}=.979)$, del cual se puede asumir que sus actitudes y sus recursos para responder en este campo son semejantes.

\section{Contrastación de hipótesis}

$\mathrm{Al}$ analizar la segunda hipótesis (H2), en la que se compara la variable Adaptación de Conducta en ambas muestras de estudio, se obtiene un coeficiente $t=-3.177$, lo que demuestra que existen diferencias estadísticamente significativas, por lo que se acepta la segunda hipótesis.

Tabla 6. Prueba t de la variable Adaptación de Conducta, entre escolares de colegios estatales y particulares.

\begin{tabular}{|l|c|c|}
\hline \multicolumn{1}{|c|}{ Factores } & $\mathbf{t}$ & $\begin{array}{c}\text { Diferencia } \\
\text { de medias }\end{array}$ \\
\hline Personal & $-3.775 *$ & -1.85 \\
\hline Familiar & $-3.476 *$ & -1.89 \\
\hline Escolar & .979 & .55 \\
\hline Social & $-3.804 *$ & -1.68 \\
\hline Total Adaptación de conducta & $-3.177 *$ & -4.88 \\
\hline
\end{tabular}

$\mathrm{p}<.05$ 


\section{c.2. Análisis de las comparaciones, según la variable depresión, en escolares de colegios estatales y particulares (Tabla 7).}

Desde el punto de vista del puntaje total, no se encuentra diferencia estadísticamente significativa $(\mathrm{r}=-.138)$, sin embargo, al realizar las comparaciones a través de los subtests de la prueba, se obtienen los siguientes resultados:

- Sí, se observan diferencias estadísticamente significativas en los subtests Respuesta Afectiva, Problemas Sociales, Autoestima, Preocupación por la muerte, Sentimiento de Culpa y Depresivos varios.

- No se observan diferencias estadísticamente significativas en los subtests de reactivos positivos Ánimo - Alegría y Positivos Varios.

- Los escolares de colegios estatales alcanzan un mayor puntaje en la variable Depresión. Se puede decir que son más vulnerables hacia la depresión.

\section{Contrastación de hipótesis}

Al analizar la tercera hipótesis (H3), referida a los factores de la depresión, entre escolares de colegios estatales y particulares, se obtuvo como resultado un puntaje $t$ $=-.138$, en la que no se observan diferencias estadísticamente significativas, desde el punto de vista de sus puntajes totales, por lo tanto no se acepta la tercera hipótesis.

Tabla 7. Prueba t de comparación de los factores de la depresión entre escolares de colegios estatales y particulares.

\begin{tabular}{|l|c|c|}
\hline \multicolumn{1}{|c|}{ Factores } & t & $\begin{array}{c}\text { Diferencia } \\
\text { de medias }\end{array}$ \\
\hline Ánimo - Alegría & -.118 & -.05 \\
\hline Respuesta afectiva & $5.734 *$ & 3.48 \\
\hline Problemas sociales & $4.187 *$ & 2.62 \\
\hline Autoestima & $4.839 *$ & 2.83 \\
\hline Preocupación por la muerte & $3.851 *$ & 1.92 \\
\hline Sentimiento de culpa & $5.609 *$ & 3.19 \\
\hline Depresivos varios & $5.289 *$ & 3.00 \\
\hline Positivos varios & -.126 & -.07 \\
\hline Total depresivo & -.138 & -.12 \\
\hline
\end{tabular}

$\mathrm{p}<.05$ 


\section{d) Análisis comparativo en función al género y tipo de colegio}

d.1. Análisis de las comparaciones, según la variable adaptación de conducta, en escolares de colegios estatales, según género (Tabla 8).

No se observan diferencias estadísticamente significativas, tanto al tomar en cuenta los puntajes totales de la prueba, como los resultados parciales de la misma, en lo que se refiere a los subtests.

De esto se puede interpretar que los roles que asumen en las diferentes áreas, es igual tanto en varones como en mujeres, motivo por el cual no se observan diferencias significativas.

Tabla 8. Prueba t de comparación de los factores de la adaptación de conducta en escolares de colegios estatales, según género.

\begin{tabular}{|l|c|c|}
\hline \multicolumn{1}{|c|}{ Factores } & $\mathbf{t}$ & $\begin{array}{c}\text { Diferencia } \\
\text { de medias }\end{array}$ \\
\hline Personal & .476 & .27 \\
\hline Familiar & 2.311 & 1.49 \\
\hline Escolar & -1.303 & -.86 \\
\hline Social & -.572 & -.30 \\
\hline Total adaptación de conducta & .332 & .60 \\
\hline
\end{tabular}

d.2. Análisis de las comparaciones según la variable adaptación de conducta, en escolares de colegios particulares, según género (Tabla 9).

No se observan diferencias estadísticamente significativas, al tomar en cuenta los puntajes totales de la prueba, pero sí se observa una diferencia significativa en el subtest Adaptación Familiar.

Estos resultados permiten interpretar que los roles son más marcados en el ámbito familiar, en lo que respecta a los varones que a las mujeres.

Tabla 9. Prueba t de comparación de los factores de la adaptación de conducta en escolares de colegios particulares, según género.

\begin{tabular}{|l|c|c|}
\hline \multicolumn{1}{|c|}{ Factores } & t & $\begin{array}{c}\text { Diferencia } \\
\text { de medias }\end{array}$ \\
\hline Personal & 2.293 & 1.92 \\
\hline Familiar & $4.268 *$ & 3.63 \\
\hline Escolar & -.423 & -.40 \\
\hline Social & .371 & .27 \\
\hline Total adaptación de conducta & $\mathbf{2 . 1 0 8}$ & $\mathbf{5 . 4 2}$ \\
\hline
\end{tabular}

$\mathrm{p}<.05$ 


\section{Contrastación de hipótesis}

$\mathrm{Al}$ analizar la cuarta hipótesis (H4), desde el punto de vista de los puntajes totales, no se encuentran diferencias estadísticamente significativas, tanto en los escolares de colegios estatales como en los particulares, obteniéndose un puntaje $\mathrm{t}=.332 \mathrm{y} \mathrm{t}=$ 2.108 respectivamente, por lo que no se acepta la cuarta hipótesis.

\section{d.3. Análisis de las comparaciones según la variable Depresión, en escolares de colegios} estatales, según género (Tabla 10).

No se observan diferencias estadísticamente significativas, al tomar en cuenta los puntajes totales de la prueba como al analizar los resultados parciales de la misma.

De esto se puede interpretar que los roles que asumen los varones y las mujeres son semejantes y con el mismo nivel de vulnerabilidad para deprimirse.

Tabla 10. Prueba t de comparación de los factores de depresión en escolares de colegios estatales, según género.

\begin{tabular}{|l|c|c|}
\hline \multicolumn{1}{|c|}{ Factores } & $\mathbf{t}$ & $\begin{array}{c}\text { Diferencia } \\
\text { de medias }\end{array}$ \\
\hline Ánimo - Alegría & -1.785 & -.96 \\
\hline Respuesta afectiva & -2.497 & -1.84 \\
\hline Problemas sociales & -1.505 & -1.13 \\
\hline Autoestima & -.623 & -.44 \\
\hline Preocupación por la muerte & -.164 & -.10 \\
\hline Sentimiento de culpa & .281 & .19 \\
\hline Depresivos varios & -2.528 & -1.67 \\
\hline Positivos varios & -.235 & $\mathbf{- . 1 6}$ \\
\hline Total depresivo & $\mathbf{- 1 . 0 3 3}$ & $\mathbf{- 1 . 1 2}$ \\
\hline
\end{tabular}

\section{d.4. Análisis de las comparaciones según la variable depresión, en escolares de colegios particulares, según género (Tabla 11).}

No se observan diferencias estadísticamente significativas, al tomar en cuenta los puntajes totales de la prueba. Pero sí se observan diferencias significativas en el subtest Depresivos Varios.

Se puede entender que existe ligeramente una mayor vulnerabilidad de parte de la mujer en el campo de la depresión. 
Tabla 11. Prueba t de comparación de los factores de depresión, en escolares de colegios particulares, según género.

\begin{tabular}{|l|c|c|}
\hline \multicolumn{1}{|c|}{ Factores } & t & $\begin{array}{c}\text { Diferencia } \\
\text { de medias }\end{array}$ \\
\hline Ánimo - Alegría & -2.080 & -1.34 \\
\hline Respuesta afectiva & -2.582 & -2.41 \\
\hline Problemas sociales & -2.526 & -2.49 \\
\hline Autoestima & -2.125 & -1.97 \\
\hline Preocupación por la muerte & -.848 & -.71 \\
\hline Sentimiento de culpa & -.602 & -.58 \\
\hline Depresivos varios & $-3.226 *$ & -2.97 \\
\hline Positivos varios & -1.839 & -1.39 \\
\hline Total depresivo & -2.167 & -2.74 \\
\hline
\end{tabular}

$\mathrm{p}<.05$

\section{Contrastación de hipótesis}

Al analizar la quinta hipótesis (H5), y desde el punto de vista de los puntajes totales, no se encuentran diferencias estadísticamente significativas, tanto en los escolares de colegios estatales como en los particulares, obteniéndose un puntaje $\mathrm{t}=-1.033 \mathrm{y} \mathrm{t}=$ -2.167 respectivamente, por lo que no se acepta la quinta hipótesis.

\section{DISCUSIÓN DE LOS RESULTADOS}

En lo que respecta al análisis de las pruebas, Adaptación de Conducta y Depresión, nos permiten afirmar que los resultados del análisis de ítems, especialmente de la primera prueba, Adaptación de Conducta, el cual mide los niveles de ajuste psicosocial, se obtienen valores significativos, por lo que se puede concluir que todos los ítems son relevantes en la estructuración de dicho instrumento, permitiéndonos con esto obtener datos confiables.

Sin embargo, en la segunda prueba de Depresión, se tuvieron que eliminar algunos ítems (catorce ítems), que fueron los que tuvieron un valor negativo, tal como se recomienda en los procedimientos psicométricos para este caso, y que se puede ver en el anexo correspondiente. Al ser depurados los ítems que quedaron, sí alcanzaron un valor significativo y relevante para la estructuración de la prueba, y por lo tanto, confiables en sus resultados.

Del análisis de las correlaciones, y tomando como referencia ambas variables, Adaptación de Conducta y Depresión, así como las muestras de estudio (estatal y particular), se obtuvo una correlación significativa, sin embargo, al realizar el análisis de los subtest correspondientes, se observó que mientras que para los escolares de colegio estatal, la 
adaptación social no tenía implicancias en las respuestas afectivas y problemas sociales, sin embargo, en los escolares de colegios particulares, sí tenía implicancias en esos mismos aspectos, vale decir que son más susceptibles de responder afectivamente y expresar problemas sociales, como manifestación de su ajuste psicosocial.

En el análisis de las comparaciones, y tomando como referencia la variables Adaptación de Conducta, se observó diferencias significativas, con un puntaje más favorable en los escolares de colegios particulares. Las diferencias más significativas fueron en los subtest Adaptación Personal, Adaptación Familiar y Adaptación Social, por lo que se asume que los escolares de colegios estatales enfrentan una mayor dificultad en estas áreas.

No se encuentra diferencias significativas entre ambas muestras con relación a la variables Depresión, pero sí en algunos subtest de la prueba, como por ejemplo en los subtest Respuesta Afectiva, Problemas Sociales, Autoestima, Preocupación por la muerte, Sentimiento de Culpa y Depresivos varios, con un puntaje mayor en los escolares de colegios estatales.

Tomando como referencia el género, el tipo de colegio y la variables Adaptación de Conducta, no se encontraron diferencias significativas entre varones y mujeres de colegios estatales y particulares. Pero en estos últimos sí se encontró diferencia significativa en el subtest Adaptación Familiar, por lo que se asume que los roles entre el hombre y la mujer son más marcado en esta muestra en relación a los escolares de colegios estatales.

Tomando como referencia el género, el tipo de colegio y la variables Depresión, no se encontraron diferencias significativas entre varones y mujeres de colegios estatales y particulares. Pero en éstos últimos, sí se encontró diferencia significativa en el subtest Depresivos varios, por lo que se asume una mayor susceptibilidad hacia la depresión en los escolares mujeres, de esta muestra de estudio.

De todo lo anteriormente presentado, es recomendable realizar una indagación mayor, tomando como referencia estas mismas variables, pero en escolares de menos edad, espacialmente de los grados de primaria.

\section{CONCLUSIONES}

1. Existe una correlación significativa entre la variable Ajuste Psicosocial y la variable Depresión, tanto en escolares de centros escolares estatales como en particulares de Lima Metropolitana.

2. Existen diferencias estadísticamente significativas en la variable Ajuste Psicosocial entre escolares de colegios estatales y particulares.

3. No existen diferencias estadísticamente significativas en la variable Ajuste Psicosocial entre escolares de colegios estatales según género.

4. No existen diferencias estadísticamente significativas en la variable Depresión entre escolares de colegios estatales y particulares, pero sí al interior de sus factores: Respuesta afectiva, problemas sociales, autoestima, preocupación por la muerte, sentimiento de culpa y depresivos varios. 
5. No existen diferencias estadísticamente significativas en la variable Ajuste Psicosocial, en escolares de centros escolares particulares según género, pero sí en el subtest adaptación familiar (mayor en varones).

6. No existen diferencias estadísticamente significativas en la variable Depresión, en escolares de colegios estatales según género.

7. No existen diferencias estadísticamente significativas en la variable Depresión, en escolares de colegios particulares según género, salvo en el subtest depresivos varios (mayor en mujeres).

\section{REFERENCIAS BIBLIOGRÁFICAS}

1. Saldaña, C. (2001). Detección y prevención en el aula de los problemas del adolescente. Madrid: Ediciones Pirámide.

2. Ponce, C. (2003). «Conducta antisocial-delictiva y satisfacción familiar en un grupo de estudiantes de quinto de secundaria de Lima Metropolitana, pertenecientes a diferentes estratos socioeconómicos». Revista de investigación en psicología. Lima: Instituto de Investigaciones Psicológicas de la UNMSM. Vol. 6, Nº 1, pp. 104-125.

3. Berry, J. (1992). Cultura y salud mental, Seminario de Post Grado. Buenos Aires: Facultad de Psicología de la Universidad de Buenos Aires.

4. Arenas, C. (1998). «Los rostros escondidos y la acción social frente a la marginalidad: Violencia juvenil a fines de los 90». Revista de psicología. Lima: Facultad de Psicología de la UNMSM. Vol. II, Nº 1, pp. 97-98.

5. Casullo, M., Bonaldi, P., y Fernández, M. (2000). Comportamientos suicidas en la adolescencia. Morir antes de la muerte. Buenos Aires: Lugar Ed.

6. Sánchez, H., y Reyes, C. (2002). Metodología y diseños en la investigación científica. Lima: Editorial Universitaria de la URP.

7. De la Cruz, M., y Cordero, A. (1981). Inventario de adaptación de conducta. Madrid: Editorial TEA.

8. Lang, M., y Tisher, M. (1990). Cuestionario de depresión para niños. Madrid: Editorial TEA. 